\title{
Das Preferências de Jogo à Criação do Mobile Game Konnecting: um estudo no ensino superior
}

\author{
Ana Amélia Carvalho ${ }^{1}$, Inês Araújoํㅜ André Fonseca ${ }^{1}$ \\ anaameliac@fpce.uc.pt, inesaraujo@fpce.uc.pt, afcfonseca93@gmail.com \\ ${ }^{1}$ Faculdade de Psicologia e de Ciências da Educação da Universidade de Coimbra, Rua do Colégio Novo, \\ 3000-115 Coimbra, Portugal
}

DOI: 10.17013/risti.16.30-45

\begin{abstract}
Resumo: Tendo presente as potencialidades dos jogos para aprender e o interesse crescente dos estudantes por jogar, desenvolvemos um jogo mobile para estudantes de licenciatura, começando por conhecer as suas preferências de jogo. Neste artigo apresentam-se as etapas conducentes à criação do jogo, nomeadamente: os jogos que os estudantes universitários mais gostam de jogar, tendo-se realizado um survey no ensino superior $(\mathrm{n}=626)$. De seguida, procedeu-se à análise desses jogos, tendo por base os 36 princípios de aprendizagem propostos por Paul Gee, bem como as mecânicas de jogo identificadas por Ernest Adams e Joris Dormans. Por fim, descreve-se o jogo Konnecting, explicitando-se as mecânicas de jogo, e os princípios de aprendizagem subjacentes.
\end{abstract}

Palavras-chave: Ensino Superior; Preferências de Jogo; Mobile game; Princípios de aprendizagem; Konnecting.

\section{From game preferences to the creation of Mobile Game Konnecting: a study in higher education}

\begin{abstract}
Keeping in mind the advantages of using games for learning and the increasing interest of students to play games, we developed a mobile game for undergraduate students. The starting point was to first know their preferences. In this paper, the steps that lead to the creation of the game will be presented, namely: the games that university students most enjoy playing, collected through a survey carried out in higher education $(n=626)$; further, these games were analyzed according to the learning principles suggested by Paul Gee, as well as the game mechanics identified by Ernest Adams e Joris Dormans. Finally, the game Konnecting is described, explaining the mechanics involved and the underlying learning principles.
\end{abstract}

Keywords: Higher Education; Game preferences; Mobile game; Learning principles, Konnecting. 


\section{Introdução}

Apresentamos o jogo Konnecting: o Homem ser comunicante, sobre a evolução da comunicação humana, que teve subjacente um estudo sobre as preferências de jogo mobile dos estudantes universitários portugueses. O jogo foi desenvolvido para o sistema Android, dado ser o sistema operativo mais popular em Portugal (APDC, 2013), pretendendo envolver os estudantes na aprendizagem através de pequenas tarefas temporizadas.

O aumento da procura pela formação no ensino superior, trouxe estudantes com preparação e interesses diversificados, habituados a um acesso constante à informação online e frequentemente conectados à rede e aos amigos. Estes estudantes, designados por uns como nativos digitais (Prensky, 2010) e por outros como superficiais (Carr, 2012), são inseparáveis dos seus dispositivos móveis, como se fossem uma extensão da sua pessoa.

Rentabilizar os dispositivos móveis dos estudantes nas aulas pode contribuir para que o professor consiga ter alunos mais envolvidos e ativos na aprendizagem, como é o caso de respostas eletrónicas (Student Response System), de quizzes ou de sondagens durante a dinâmica da aula (Carvalho, 2012; Oliveira, 2014).

Se, por um lado, temos que identificar os conhecimentos prévios dos estudantes (Laurillard, 2002), por outro, temos que nos adaptar ao seu perfil interativo e conectado, para os motivarmos para a aprendizagem no ensino superior. Para a geração atual os jogos fazem parte da sua vida desde que nascem, estes são um espaço muito mais cativante que a realidade, onde podem ser um herói invencível, um guerreiro destemido, solucionar problemas sem fim... Os jogadores sentem-se invencíveis, competentes, motivados e a sua auto-estima eleva-se, é por isso que investem o tempo que podem nesta atividade abdicando da realidade que não os estimula (McGonigal, 2011). A integração de jogos mobile pode ser, assim, uma forma de os cativarmos. Tal como defendem Hamari \& Nousiainen (2015) "[t]he aim of these developments in educational context is to promote student engagement and consequently positively influence learning outcomes" (p.1)

O presente artigo aborda as aportações dos jogos na aprendizagem, passando depois a centrar-se no estudo realizado com estudantes do ensino superior, sobre as suas preferências de jogo, os jogos mais jogados e a análise feita com base nos 36 princípios de aprendizagem propostos por Gee (2003). Por fim, sintetizam-se os componentes que os jogos para o ensino superior podem ter, fruto das preferências recolhidas, e que implementamos no jogo Konnecting.

\section{Jogar e Aprender}

O ser humano possui na sua essência a necessidade de aprender e assim conseguir superar os desafios da sobrevivência. A cada conquista o ser humano experiencia a sensação de superação, de conquista, o que lhe dá prazer. Aprender é assim uma fonte de prazer, sendo que é através desta que o jogo proporciona a sensação de fun (Koster, 2005). Como salienta Huizinga (2000), jogar e o brincar são uma atividade intrinsecamente humana, que permite o seu desenvolvimento cultural e cognitivo. 
Os jogos ganharam um novo impacto com o desenvolvimento tecnológico, captando cada vez mais a atenção das novas gerações (Malliet \& Meyer, 2005). Hoje em dia quase a totalidade dos jovens que entram no ensino superior jogam ou experienciaram um qualquer videojogo, estão habituados a uma tecnologia que lhes proporciona vivências e experiências muito mais aliciantes que a realidade que os circunda (McGonigal, 2011). Como poderá o ensino acompanhar as exigências de uma geração cada vez mais estimulada pelas tecnologias digitais? São aspetos que urge investigar.

Os jogos são constituídos por regras, rotinas, ações, feedback, objetivos, conflito e interação social (Gee, 2007; Klopfer, 2008; Prensky, 2010; Squire, 2011). Exigem do jogador ações como a resolução de problemas, a tomada de decisão, a capacidade de saber lidar com o fracasso e o sucesso. Proporcionam ao jogador o desenvolvimento de capacidades como a persistência, bem como de destrezas cognitivas e motoras necessárias para obterem sucesso no jogo e na vida.

O jogo envolve o jogador, como referem Salen \& Zimmerman (2004): "a game is a system in which players engage in an artificial conflict, defined by rules, that results in a quantifiable outcome"(p. 80).

Tal como o brincar, a que todos os seres vivos recorrem para experimentar o mundo e aprender (Huizinga, 2000), também o jogo possibilita recriar num ambiente seguro e o mais variado tipo de experiências humanas ou de fantasia (Kapp, 2012).

Ao contrário da vida real, no jogo é possível repetir as vezes que forem necessárias, aprendendo com cada uma e evoluindo até alcançar o objetivo proposto. Tal como salienta Gee (2003), o jogo proporciona um significado para que se aplique tempo e esforço na execução de uma atividade, sempre num ambiente seguro, onde as consequências não são reais e onde é possível testar hipóteses, sem receios.

Mas os jogos não são sistemas fechados em si mesmos, acompanham cada jogador para a própria realidade, através da interação de diferentes jogadores que possuem o mesmo jogo como alvo dos seus interesses. Com as atuais possibilidades de interação social que as novas tecnologias proporcionam é fácil encontrar comunidades de centenas, senão milhares de jogadores onde, de modo informal, se partilham conquistas, questões e experiências. "James Paul Gee, who coined the term "affinity spaces", noted how they form through voluntary associations (i.e., we all are there by choice) and sharing of knowledge and expertise. Some, but not all, of these affinity spaces can be described as communities" (Squire, 2011, p. 64).

Para Gee (2003) todas estas características conferem aos jogos um exemplo de aprendizagem, tendo identificado princípios de aprendizagem que no seu entender a escola deveria assimilar para possibilitar uma aprendizagem efetiva.

Connolly, Boyle, MacArthur, Hainey \& Boyle (2012) ao realizarem uma revisão da literatura sobre os jogos verificaram que "playing computer games is linked to a range of perceptual, cognitive, behavioral, affective and motivational impacts and outcomes. The most frequently occurring outcomes and impacts were knowledge acquisition/content understanding and affective and motivational outcomes” (p. 661). É de salientar que o efeito principal nos jogadores incide principalmente na aquisição de competências e na compreensão de conteúdo, objetivos semelhantes ao ensino formal. 
Tal como para Gee (2003), também para Hamari et al. (2016): os jogadores sentem-se motivados para aprender, em parte, porque a aprendizagem é situada e ocorre através de um processo de colocar hipóteses, experimentar e refletir sobre o mundo do jogo. Além disso, os objetivos são claros e a informação é apresentada à medida que é necessária para se atingir cada objetivo. Ao contrário de muitas tarefas escolares, os jogos criam um contexto com objetivos claros, onde o esforço tem significado.

Ao utilizar jogos para fomentar a aprendizagem, estamos a envolver os alunos na resolução de problemas e num trabalho de projeto, o que permite que os estudantes "see more connections, becoming more intrinsically interested, and thus also pay better attention” (Hamari et al., 2016, p. 171). Ao dar significado às tarefas, o esforço que o aluno está disposto a realizar é muito maior, pois o aluno sabe que o que está a fazer irá ser útil para algo.

Ao analisar o impacto de fatores como o envolvimento (engagement), imersão (immersion), desafio (challenge) e capacidades percebidas (perceived skill) na aprendizagem, Hamari et al. (2016) concluíram que o envolvimento no jogo tem um efeito positivo na aprendizagem Por outro lado, verificaram que a imersão no jogo não tem um efeito significativo. "The perceived challenge of the game affected learning, both directly and via the increased engagement. Challenge was an especially strong predictor of learning outcomes. Perceived skill did not affect learning directly, but it also impacted learning via a significant mediation effect through engagement” (idem, p. 175). É de salientar que o envolvimento do jogador no jogo, o desafio do jogo e o que é percebido pelo jogador são importantes como preditores de aprendizagem. Se para atingir o nível de flow - ou experiência ótima, "estado de concentração profunda" (Csikszentmihalyi, 2002, p. 69) - é necessário que seja suficientemente desafiante, mas sem ser demasiado fácil. Importa, então, criar um jogo que mantenha um equilíbrio constante entre o desafio que proporciona e a capacidade do jogador em realizá-lo.

Os jogos, com a designação de serious game ou de game-based learning, têm vindo a ser utilizados no ensino superior (Fitz-Walter, Tjondronegoro, \& Wyeth, 2012; Poy-Castro, Mendaña-Cuervo, \& González, 2015; Whitton, 2009, 2010; Zolotov, 2014). Whitton (2010) ao reconhecer os jogos como uma excelente ferramenta de aprendizagem para adultos, salienta que deveremos, no momento de os aplicar, ter o cuidado de esclarecer o contexto e os objetivos de sua aplicação, sendo estes mais eficazes quando estão diretamente ligados à prática.

É neste contexto que importa caracterizar os destinatários de jogos mobile quando se pretende desenvolver jogos. Só conhecendo as preferências e hábitos dos jogadores se podem desenhar jogos mais envolventes e que possam criar o estado de flow necessário.

\section{Preferências de Jogo Mobile no Ensino Superior}

Para concebermos o jogo Konnecting, e no âmbito do projeto "Dos Jogos às Atividades Interativas para Mobile-Learning”, consideramos necessário conhecer as preferências de jogo mobile dos estudantes universitários, tendo subjacente a seguinte questão de investigação: Que tipos de jogos preferem os estudantes? 
Desenvolvemos um questionário com quatro dimensões, nomeadamente: 1) Caracterização do estudante, 2) Caracterização dos hábitos de jogo, 3) Preferências sobre os jogos e 4) Aprendizagem de conteúdos curriculares através de jogos. O instrumento foi avaliado por três peritos em jogos e em metodologia da investigação.

O survey (Babbie, 1997) foi realizado online no ensino superior de 21 de maio a 16 de setembro de 2013, em Portugal. Responderam 1101 estudantes, 693 sujeitos do género feminino e 408 do género masculino, sendo que destes apenas 626 são jogadores, isto é, $56,9 \%$ (Tabela 1 ).

\begin{tabular}{lll}
\hline Género & $\begin{array}{l}\text { Amostra } \\
\text { f }\end{array}$ & $\mathbf{\%}$ \\
\hline Feminino & 363 & 58,0 \\
Masculino & 263 & 42,0 \\
\hline Total & 626 & 100,0 \\
\hline
\end{tabular}

Tabela 1 - Género da amostra

A maioria dos sujeitos da amostra é do género feminino (58\%), tal como a maioria dos estudantes no ensino superior em Portugal (cf. PORDATA ${ }^{1}$ de 2013). Relativamente aos ciclos de estudos, verifica-se que a maioria é do $1^{\circ}$ Ciclo de estudos $(65,3 \%)$, tal como ocorre com o número de estudantes matriculados no país por ciclos (231.468 no $1^{\circ}$ Ciclo e 117.265 no $2^{\circ}$ Ciclo de estudos). A maioria dos alunos tem menos de 23 anos (70,8\%) o que se enquadra na idade média de frequência dos $1^{\circ}$ e $2^{\circ}$ Ciclos.

\subsection{Resultados do Survey no Ensino Superior}

O número de horas, em média, gasto por semana a jogar é no total de 4,2h, mas são eles que jogam mais tempo $(5,4 \mathrm{~h})$, elas ficam pelas $3,4 \mathrm{~h}$.

A maioria dos respondentes $(71,6 \%)$ prefere jogar só, embora se distribuam do seguinte modo: $82,1 \%$ para o género feminino e 57,0\% para o género masculino (Tabela 2). Os que preferem jogar com outros online $(28,4 \%)$ fazem-no sobretudo em equipas $(18,2 \%)$ e são sobretudo jogadores do género masculino (43,3\%).

\begin{tabular}{llll}
\hline Jogar jogos & $\begin{array}{l}\text { Total } \\
\mathbf{\%}\end{array}$ & $\begin{array}{l}\text { Feminino } \\
\mathbf{\%}\end{array}$ & $\begin{array}{l}\text { Masculino } \\
\mathbf{\%}\end{array}$ \\
\hline Só & 71,6 & 82,1 & 57,0 \\
Com outros online & 28,4 & 17,9 & 43,0 \\
$\quad$ Em equipa & 18,2 & 7,4 & 33,1 \\
Um contra outro & 13,3 & 9,6 & 18,3 \\
\hline
\end{tabular}

Tabela 2 - Preferência por jogar só ou acompanhado

\footnotetext{
${ }^{1}$ Informação em: http://www.pordata.pt/Portugal/Alunos+matriculados+pela+1.a+vez+no+ensino+ superior+total+e+por+sexo-1047
} 
Os respondentes referiram um total de 177 jogos, mas os cinco jogos mais jogados são Candy Crash, Angry Birds, The Sims, Bubbles, e na $5^{\mathrm{a}}$ posição surgem em ex-aequo: Flow, Fruit Ninja e Paciência (Tabela 3).

\begin{tabular}{llll}
\hline $\begin{array}{l}\text { Ordenação dos } \mathbf{5} \\
\text { jogos mais jogados }\end{array}$ & Todos os sujeitos & Feminino & Masculino \\
\hline $1^{o}$ & Candy Crash & Candy Crash & Angry Birds \\
$2^{o}$ & Angry Birds & The Sims & League of Legends (LoL) \\
$3^{o}$ & The Sims & Angry Birds & Football Manager (FM) \\
$4^{o}$ & Bubbles & Bubbles & $\begin{array}{l}\text { Pro Evolution Soccer } \\
\text { (PES) }\end{array}$ \\
& $\begin{array}{l}\text { Flow / Fruit Ninja / } \\
5^{o}\end{array}$ & Fruit Ninja & Flow / Hill Climb Racing \\
\hline
\end{tabular}

Tabela 3 - Jogos mais jogados pelo total dos respondentes e por género

Na altura em que este estudo foi realizado, o Candy Crash Saga era o jogo mais popular entre os estudantes universitários em Portugal (Carvalho et al., 2014), mas também refletia uma tendência generalizada. Foi o jogo que obteve mais downloads em 2013 na iOS App Store e no Google Play (App Annie, 2014). Também se constatou que quatro dos jogos mais jogados pelos estudantes portugueses pertencem ao ranking mundial: Candy Crash Saga, Angry Birds, Fruit Ninja e Hill Climb Racing.

Analisando as preferências por género, verifica-se que os sujeitos do género masculino preferem jogos de desporto, nomeadamente futebol (PES e $F M$ ). São jogos longos onde são necessários objetivos bem definidos e alcançáveis através de esforço (LoL, PES e FM). Eles gostam de interagir com sistemas, ou seja, um conjunto definido de estruturas, comportamentos e interações com limites específicos de tempo e de espaço (Flow e Hill Climb Racing). Já os sujeitos do género feminino preferem jogos com partidas curtas e sistemas de recompensa elevados. O objetivo não é atingir o fim, mas ser reconhecido entre os pares, recebendo elogios do sistema, partilháveis com os pares, enquanto se joga (Candy Crush, Angry Birds, Bubbles e Fruit Ninja) (Carvalho, Araújo \& Zagalo, 2014).

\section{Análise dos Jogos Mais Jogados \\ 4.1. Características do Jogo consideradas mais importantes}

Inquirimos os estudantes sobre quais são as características de um jogo que eles consideram mais importantes para continuarem a jogar, tendo presente o nosso objetivo de criar um jogo.

Na Figura 1, verifica-se que se destaca, como a característica mais pontuada do jogo o gameplay, seguindo-se os cenários, os efeitos gráficos e as animações, a história, as personagens, ser um jogo com muitos níveis e ser um jogo longo. Os sons e a música são os menos considerados. Squire (2011) também considera que não é de admirar dada a familiaridade dos jogadores com essas características. 


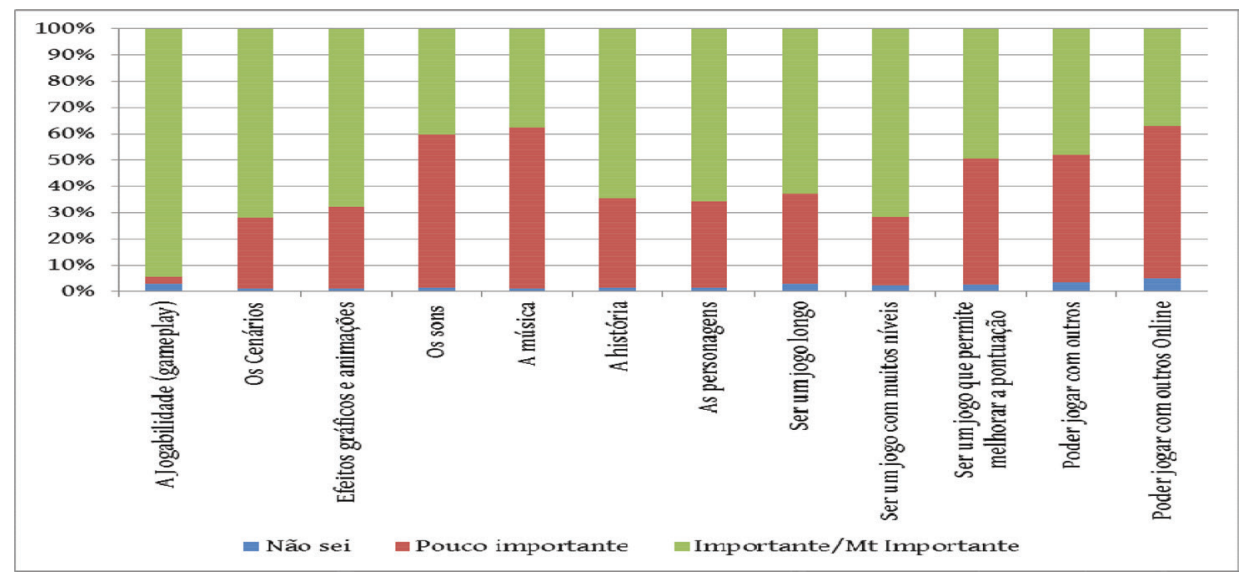

Figura 1 - Importância das características do jogo para continuar a jogar

No instrumento de recolha de dados, também se perguntava porque gostavam de jogar esses jogos. Para a análise das respostas, baseamo-nos nas seis dimensões identificadas por Sherry, Lucas, Greenberg, \& Lachlan (2006): Excitação, Desafio, Competição, Desvio, Fantasia e Interação social. Mas rapidamente nos apercebemos que não eram suficientes para classificar as respostas, tendo incluído mais duas dimensões: Características específicas do jogo (nomeadamente as características do game design: música, cenários, gráficos, ações, história, recompensas, tipo de jogo, etc.) e a Aprendizagem (se o respondente destaca que aprende alguma coisa). Na Figura 2, destacam-se como motivos para jogar os jogos as Características específicas do Jogo (45,5\%), o Desafio (31,3\%) e a Fantasia (20,0\%). Como características específicas mais mencionadas identificámos o tipo de jogo, os efeitos gráficos e os cenários. Já relativamente ao desafio o que é mais mencionado são capacidades específicas que o jogo exige, em termos de fantasia mencionam a temática do jogo (principalmente futebol) e ações que podem realizar (e.g., ser treinador, criar histórias).

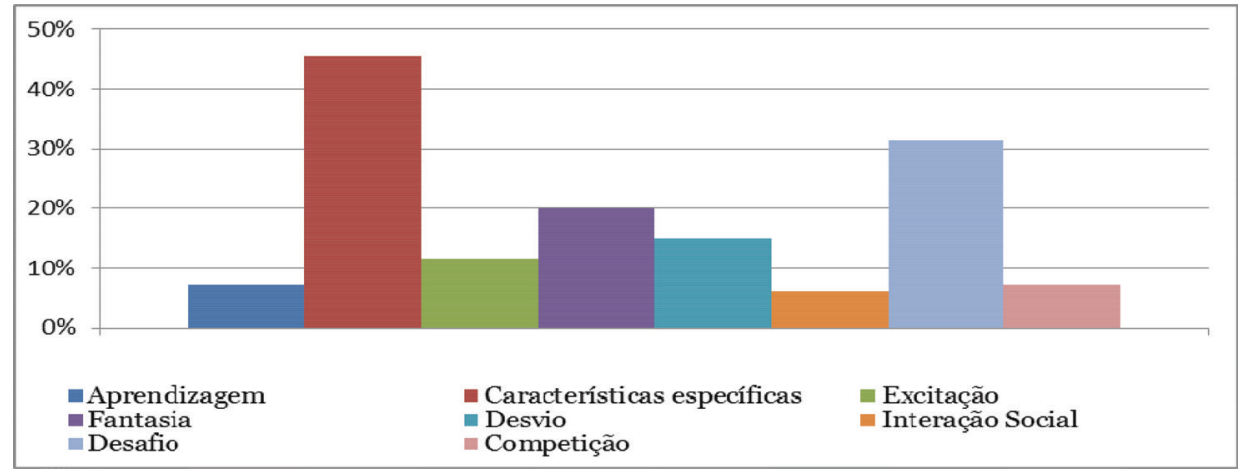

Figura 2 - Motivos para gostar de jogar os jogos 
É importante recordar, como já foi mencionado, que no estudo realizado por Hamari et al. (2016), o desafio que o jogo proporciona é um bom preditor da aprendizagem. Pelos dados que recolhemos, o desafio sentido é um dos componentes que mais cativam os estudantes do Ensino Superior em Portugal.

\subsection{Aprender através de Jogos no Ensino Superior}

Por fim, sondamos os estudantes sobre se gostariam de jogar jogos como complemento às suas atividades de aprendizagem. A maioria respondeu favoravelmente $(78,12 \%)$, verificando-se uma semelhança entre os géneros, elas manifestando a sua concordância com 78,5\% e eles com 77,6\%. Aos que responderam afirmativamente solicitamos que nos indicassem o tipo de jogo que gostariam de jogar para aprender os conteúdos curriculares, tendo por base a lista apresentada por Connolly et al. (2012): Ação, Aventura, Desporto, Estratégia, First Person Shooter, Casual games, Jogos de Corridas, Board Game, Luta, Plataforma, Puzzle, Role-Playing e Simulação.

Na Tabela 4, apresentam-se os respondentes $(n=489)$ que são favoráveis à utilização de jogos para aprender os conteúdos curriculares. Os tipos de jogos mais listados são de estratégia (72,8\%), seguidos por simulação $(58,7 \%)$ e depois por ação (41,9\%). Esta preferência geral também se reflete na mesma ordem em ambos os géneros.

\begin{tabular}{llll}
\hline Tipo de jogo & $\mathbf{N = 4 8 9}$ & $\begin{array}{l}\text { Feminino } \\
(\mathbf{n = 2 8 5})\end{array}$ & $\begin{array}{l}\text { Masculino } \\
(\mathbf{n = 2 0 4 )}\end{array}$ \\
\hline Ação & 41,9 & 35,4 & 51,0 \\
Estratégia & 72,8 & 77,5 & 66,2 \\
Simulação & 58,7 & 60,7 & 55,9 \\
\hline
\end{tabular}

Tabela 4 - Preferência pelo tipo de jogos para aprender

No entanto, os tipos de jogo indicados pelas jogadoras para aprender não correspondem aos tipos de jogo que elas jogam. Verifica-se um contraste sobretudo nas preferências das jogadoras entre o jogar por lazer versus o jogar para aprender.

\subsection{Princípios de Aprendizagem Subjacentes aos Jogos}

Depois de ter jogado vários videojogos, Gee (2003) identificou 36 princípios de aprendizagem. Os jogos mobile que os estudantes universitários jogam, na generalidade, são simples e com partidas curtas, tendo-se identificado sete princípios de aprendizagem (Tabela 5).

Os princípios de aprendizagem Psychosocial Moratorium e Amplification of Input, geralmente, estão presentes em qualquer jogo. Há a expectativa que se corram riscos sem consequências, por outro lado, tudo acontece mais rapidamente do que na vida real. Por exemplo, num jogo de futebol real uma partida dura 90 minutos enquanto que num jogo como o PES pode durar de 4 a 12 minutos.

Os seguintes três princípios Achievement, Regime of Competence e Intuitive Knowledge reportam-se ao conhecimento do jogador sobre as suas capacidades. Perante cada etapa 
superada, o jogador sente prazer, uma recompensa intrínseca, e sabe que está a dominar uma capacidade. O jogo gere o equilíbrio entre desafio e as capacidades (skills) do jogador que, com prática, vão sendo melhoradas. É esta prática que lhe dá um "intuitive knowledge" para resolver algumas tarefas, mesmo sem saber explicar como o realizou. Estes princípios levam os jogadores a sentir que são capazes de fazer qualquer coisa, que têm controlo, bastando esforço e empenhamento para o atingir.

No Concentrated Sample as regras do jogo e as possíveis ações são apresentadas no início do mesmo. Assim, elas são aprendidas rapidamente e numa fase inicial, fazendo com que o resto do jogo seja mais fácil e mais simples.

Por fim, o Affinity Group centra-se nos jogadores como elementos de um grupo que partilha um mesmo interesse, neste caso um determinado jogo. Sendo que estes grupos se podem reunir de forma presencial, embora o mais comum nos dias de hoje sejam as redes sociais e os fóruns online, onde partilham conquistas, trocam ideias sobre o próprio jogo, pedem ajuda ou ensinam os que se iniciam no jogo.

\begin{tabular}{|c|c|}
\hline Princípio & Descrição \\
\hline $\begin{array}{l}\text { 6. Psychosocial } \\
\text { Moratorium } \\
\text { Principle }\end{array}$ & $\begin{array}{l}\text { "Learners can take risks in a space where real-world consequences are lowered." } \\
\text { (p.67) }\end{array}$ \\
\hline $\begin{array}{l}\text { 10. Amplification of } \\
\text { Input Principle }\end{array}$ & "For a little input, learners get a lot of output." (p.67) \\
\hline $\begin{array}{l}\text { 11. Achievement } \\
\text { Principle }\end{array}$ & $\begin{array}{l}\text { "For learners of all levels of skill there are intrinsic rewards from the beginning, } \\
\text { customized to each learner's level, effort, and growing mastery and signaling the } \\
\text { learner's ongoing achievements." (p.67) }\end{array}$ \\
\hline $\begin{array}{l}\text { 14. Regime of } \\
\text { Competence Principle }\end{array}$ & $\begin{array}{l}\text { "The learner gets ample opportunity to operate within, but at the outer edge of, } \\
\text { his or her resources, so that at those points things are felt as challenging but not } \\
\text { "undoable." (p.71) }\end{array}$ \\
\hline $\begin{array}{l}\text { 22. Intuitive } \\
\text { Knowledge Principle }\end{array}$ & $\begin{array}{l}\text { "Intuitive or tacit knowledge built up in repeated practice and experience, often } \\
\text { in association with an affinity group, counts a great deal and is honored. Not just } \\
\text { verbal and conscious knowledge is rewarded." (p.111) }\end{array}$ \\
\hline $\begin{array}{l}\text { 25. Concentrated } \\
\text { Sample Principle }\end{array}$ & $\begin{array}{l}\text { "The learner sees, especially early on, many more instances of fundamental signs } \\
\text { and actions than would be the case in a less controlled sample. Fundamental signs } \\
\text { and actions are concentrated in the early stages so that learners get to practice } \\
\text { them often and learn them well." (p.137) }\end{array}$ \\
\hline $\begin{array}{l}\text { 35. Affinity Group } \\
\text { Principle }\end{array}$ & $\begin{array}{l}\text { "Learners constitute an "affinity group", that is, a group that is bonded primarily } \\
\text { through shared endeavors, goals, and practices and not shared race, gender, } \\
\text { nation, ethnicity, or culture." (p.212) }\end{array}$ \\
\hline
\end{tabular}

Tabela 5 - Princípios de Aprendizagem (Gee, 2003) identificados

\subsection{Mecânicas de Jogo e Game Design}

Adams \& Dormans (2012) identificam nos jogos cinco tipos de mecânicas: física, economia interna, progressão, manobra tática e interação social. Os autores identificaram para cada tipo de jogo os tipos de mecânicas que lhes estão afetas. Tendo presente essa classificação e os tipos de jogos a que correspondem os jogos mais jogados sintetizamos as mecânicas que lhes estão subjacentes. 
Os jogos mais jogados pelos estudantes correspondem aos seguintes tipos: puzzle (Candy Crash, Angry Birds, Flow e Paciência), simulação (The Sims) e arcada (Bubbles e Fruit Ninja). Os jogos puzzle integram as mecânicas de física (movimentos, disparos e salto) e de progressão (níveis pré-definidos com tarefas com dificuldade crescente, storyline para definir os objetivos dos jogadores). Os jogos de simulação comportam mecânicas de física, economia interna (power-ups, coletáveis, pontos, vidas e gestão de itens) e de progressão e os jogos de arcada, embora não apareçam na lista de Adams \& Dormans (2012), consideramos que utilizam as mecânicas de física e de progressão.

Do ponto de vista do game design, verificamos que nos jogos mais jogados, de uma maneira geral, os cenários não são reais, assemelham-se a cartoons. Os efeitos gráficos e as animações dão um feedback positivo ao jogador, dando-lhe a sensação de mestria no jogo e incentivando-o a continuar. As histórias são simples e têm como finalidade introduzir o jogador no jogo e motivá-lo a jogar (e.g., Candy Crush, Fruit Ninja e Angry Birds) ou a história vai sendo revelada ao longo do jogo, como ocorre em Bubbles. Exceção é o The Sims cuja história é mais complexa e vai sendo decidida pelo jogador, enquanto que os jogos Paciência e Flow não têm história.

As personagens são simples, de tipo cartoon, e fazem movimentos engraçados quando o jogador realiza uma jogada. Estas manifestações afetivas tendem a aumentar o envolvimento e empenho do jogador, pois acaba sempre por sentir alguma empatia por aquela personagem.

\section{Componentes a Incluir em Jogos Mobile para o Ensino Superior}

Tendo presente as preferências dos estudantes do ensino superior, os princípios de aprendizagem de Paul Gee (2003) identificados, as mecânicas de jogo propostas por Adams \& Dormans (2012) e o game design, consideramos pertinente os seguintes componentes na criação de jogo mobile para o ensino superior: o contexto, as tarefas, os níveis, o feedback, o leaderboard e o princípio do grupo de afinidade (Carvalho, Araújo \& Zagalo, 2014).

O Contexto para o desenvolvimento do jogo deve estar relacionado com a unidade curricular ou o módulo e deve refletir-se no tema da história do jogo. Os jogadores têm que resolver várias Tarefas ou missões, preferencialmente de curta duração. Essas tarefas estão relacionadas com Níveis de dificuldade ou com os diferentes temas do módulo.

Cada Tarefa tem um limite de tempo para submeter ou para completar, também pode ter um temporizador para desafiar os jogadores. O grau de dificuldade deve ser moderado mas suficientemente desafiante para manter os estudantes envolvidos, mantendo o seu interesse (Csikszentmihalyi, 2002). Pode haver mecanismos de ajuda para apoiar o jogador em dificuldade, podendo mesmo remeter para o grupo de afinidade.

As Tarefas ou missões devem ter em atenção os seguintes princípios de aprendizagem já descritos em cima: Psychosocial Moratorium, Amplification of Input, Regime of Competence, Intuitive Knowledge, Concentrated Sample e Achievement. Em síntese, permitir repetir as vezes necessárias, transmitir um sentimento de competência que, com esforço e empenho, o desafio pode ser superado e também proporcionar o sentimento de conquista a cada etapa concluída. 
O Feedback é extraordinariamente importante para aprender, porque orienta de imediato o jogador. O Feedback positivo deve ser divertido, dispondo bem o jogador. $\mathrm{O}$ efeito gráfico deve enaltecer o feito do jogador. Se as tarefas ou missões não são bem-sucedidas, o jogador recebe punições.

A execução do jogo pelo jogador bem como a sua posição relativa no mesmo face aos outros, é algo que aguça a concorrência. À medida que as tarefas vão sendo executadas podem ser visíveis no Heads-Up Display. Além disso, no Leaderboard aparece a ordenação dos jogadores, sendo visíveis apenas os cinco melhores. Se o jogador não se encontra entre estes, pode ver a sua posição. Isto permite focar a atenção dos jogadores nos primeiros lugares, sem que se sintam intimidados por atingirem lugares inferiores. Para cada Tarefa deve haver um Leaderboard diferente. Recompensas e punições são obtidas através de feedback, pontuações ou leaderboard.

Um outro aspeto que consideramos importante é o Grupo de Afinidade (Gee, 2003), que se relaciona com os aspetos sociais do grupo, permitindo a interação entre jogadores em contexto informais.

O som e a música não são muito relevantes para este nível de educação dos jogadores. Os cenários podem ser simples mas relacionados com a temática. Um tutorial e mecanismos de ajuda devem estar disponíveis.

\section{O Jogo Konnecting}

O jogo Konnecting: o Homem ser comunicante foi desenvolvido para sistema Android tendo como destinatários estudantes do ensino superior do $1^{\mathrm{o}}$ Ciclo de estudos. A descrição que se apresenta respeita os componentes identificados para criar jogos mobile.

\subsection{Contexto}

O jogo foi desenvolvido no âmbito da unidade curricular Processos de Comunicação e Educação da Licenciatura em Ciências da Educação da Universidade de Coimbra. Como o título do jogo sugere, centra-se na evolução da comunicação humana desde a Pré-História até aos nossos dias. Comostorytelling deparamo-nos com um extraterrestre - Komuniket - que é incumbido de reportar o modo como os seres terrestres comunicam (Figura 3). Na Terra, viajou no tempo e tirou várias fotografias que estão organizadas cronologicamente, mas precisa de ajuda para as compreender.

\subsection{Tarefas e níveis}

O jogo tem dois níveis: Kronos (Figuras 4 e 5), cronologia sobre a comunicação humana, dividida em sete etapas que incluem um total de 82 tarefas e Zapping temático (Figuras 6 e 7), que apresenta cinco travessias temáticas, e só é desbloqueado depois do jogador terminar o Kronos.

No Kronos, o jogador tem que identificar três palavras ou expressões que ajudem a caracterizar a imagem que lhe é apresentada (Figura 4). Para isso pode optar por ler um pequeno texto explicativo, intercetando a apresentação das três áreas de resposta. 


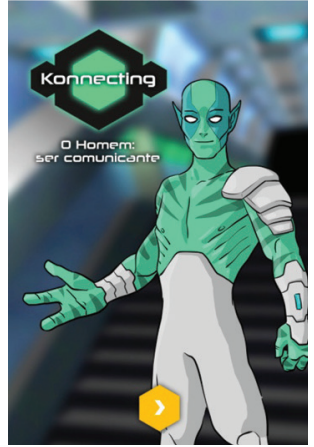

Figura 3 - O jogo Konnecting

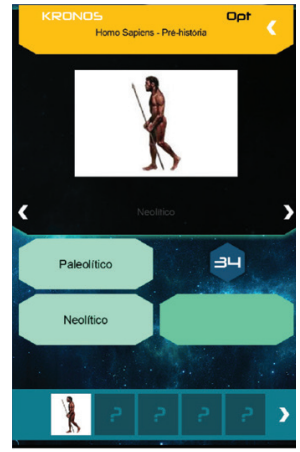

Figura 4 - Tarefa no Kronos

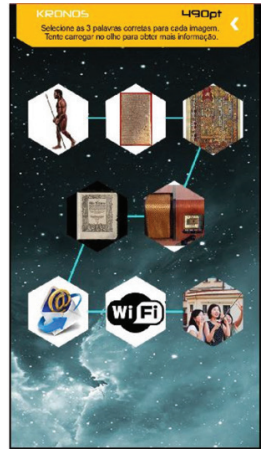

Figura 5 - Etapas do Kronos

Depois deparará com seis palavras ou expressões, onde é necessário selecionar três. Dispõe de um minuto para completar a tarefa.

O Kronos está organizado em sete etapas (Figura 5), representadas por oito imagens que correspondem aos momentos de viragem na história da comunicação, que se vão desbloqueando à medida que o jogador as executa.

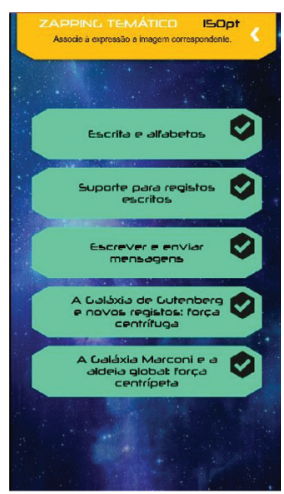

Figura 6 - Menu do Zapping temático

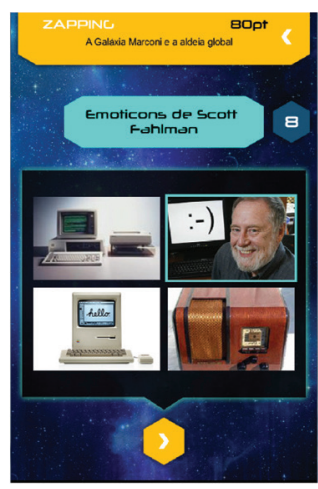

Figura 7 - Exemplo de tarefa no nível 2 - Zapping temático

No Zapping temático o jogador tem acesso a cinco travessias temáticas, como por exemplo: "Escrita e alfabetos" ou "A Galáxia Marconi e a Aldeia Global" (Figura 6). O jogador tem acesso a um pequeno texto de contextualização da temática, de seguida, perante uma expressão ou termo apresentado deve selecionar a imagem adequada, de quatro disponíveis (Figura 7).

\subsection{Feedback}

O feedback é apresentado através de efeitos gráficos e sempre que o jogador executa uma tarefa. No Kronos, perante cada imagem são validadas as três palavras selecionadas e vão 
sendo somados pontos; no Zapping temático é validada a imagem destacada, somando também pontos. Terminado o jogo, o jogador recebe do Komuniket um certificado, cujo texto depende das pontuações obtidas.

\section{4.eaderboard}

O jogo tem vários Leaderboards: total, só Kronos, só Zapping temático e de cada uma das etapas do Kronos e de cada travessia temática no Zapping. Deste modo, os jogadores, na turma onde estão registados, sabem a posição dos cinco melhores em cada tipo de leaderboard, bem como a posição relativa do próprio jogador, caso não integre os cinco primeiros. Esta informação pretende ser estimulante para o jogador; levando-o a melhorar as suas pontuações.

\subsection{Princípio do Grupo de Afinidade}

Os jogadores têm que se registar no jogo, selecionando a turma. Só podem jogar depois de aceite o seu registo pelo professor. Assim, no leaderboard sabem quem são os colegas que estão melhor posicionados.

Cria um tema de discussão que é partilhado por todos, permitindo troca de ideias, dicas, seguir as conquistas dos melhores.

\subsection{Implementação}

O jogo foi criado na linguagem de programação Java com a ajuda de uma biblioteca de desenvolvimento de jogos denominada de "libGDX". Para as animações foi também usada uma biblioteca chamada "Universal Tween Engine".

Para diminuir ao tamanho do espaço ocupado pelas imagens foram gerados atlas de imagens com a aplicação "GDX Texture Packer". Como a biblioteca "libGDX" aceita apenas fontes como imagem, foi usado o programa "BitMap Font Creator" para criar também atlas das várias fontes de texto.

O jogo tem uma componente "online", sendo os servidores utilizados os da instituição. O jogo comunica com um servidor segundo uma arquitetura "REST", enviando a informação em formato JSON.

\section{Conclusões}

O percurso feito até à criação do jogo permitiu compreender os interesses dos estudantes do ensino superior relativamente aos jogos mobile, as suas preferências de jogo, bem como a sua recetividade a aprenderem conteúdos curriculares através de jogos.

Os 36 princípios de aprendizagem propostos por Paul Gee (2003) permitiram-nos analisar os jogos mais jogados numa perspetiva pedagógica. Perante os jogos selecionados, também nos apercebemos que os estudantes têm preferência por jogos com vários níveis e partidas rápidas.

O jogo Konnecting: o Homem ser comunicante que concebemos, reflete os princípios de aprendizagem identificados, bem como as preferências de jogo dos estudantes. Apresenta 
dois níveis, mas tem muitas tarefas para serem executadas. O jogador recebe feedback frequentemente perante as suas seleções e tem um papel constantemente interventivo. Por exemplo, embora haja informação disponível, para uma mais rápida resolução da tarefa, esta não é de leitura obrigatória, devendo o jogador assinalar que a quer ler. Finalizámos os testes de usabilidade, tendo numa primeira fase sido realizados testes com um sujeito e um observador e numa segunda fase utilizou-se a técnica do inquérito por questionário (Martins, Queirós, Rocha, \& Santos, 2013). Na próxima etapa desta investigação, vamos aplicar o jogo em contexto de ensino e aquilatar a aprendizagem alcançada e a reação dos estudantes ao jogo. Esperamos, assim, atingir, conforme defendem Hamari \& Nousiainen (2015), o envolvimento dos alunos e consequentemente um efeito positivo na aprendizagem dos alunos.

\section{Agradecimentos}

Este trabalho é financiado por Fundos FEDER através do Programa Operacional Fatores de Competitividade - COMPETE e por Fundos Nacionais através da FCT - Fundação para a Ciência e a Tecnologia no âmbito do projeto - PTDC/CPE-CED/118337/2010.

\section{Referências}

Adams, E., \& Dormans, J. (2012). Game Mechanics - Advanced Game Design. Berkeley, CA: New Riders.

App Annie. (2014). App Annie Index: 2013 Retrospective - The top trends of 2013. Retrieved from http://blog.appannie.com/app-annie-index-retrospective-2013/

APDC. (2013, September 9). Smartphones crescem 25\% em Portugal. Retrieved from http://www.apdc.pt/Artigo.aspx?channel_id=004A7FFA-301B41C2-B331-1CEE7D8EAAA2\&content_id=704A96FD-oEA3-4804-848C$\mathrm{B} 7 \mathrm{C} 526679024 \& l a n g=p t$

Babbie, E. (1997). Survey Research Methods. Belmont, California: Wadsworth.

Carvalho, A. A. A. (2012). Mobile Learning: rentabilizar os dispositivos móveis dos alunos para aprender. In A. A. A. Carvalho (org.), Aprender na era digital: jogos e mobile learning (pp. 149-163). Santo Tirso: De Facto.

Carvalho, A. A., Araujo, I. C., \& Zagalo, N. (2014). A Framework for Gamified Activities Based on Mobile Games Played by Portuguese University Students. In P. Kommers, T. Issa, T. Issa, D.-F. Chang, \& P. Isaías (Eds.), Proceedings of The International Conferences On Educational Technologies (ICEduTech 2014) (pp. 89-96). New Taipei City: IADIS.

Carvalho, A. A., Araújo, I. C., Zagalo, N., Gomes, T., Barros, C., Moura, A., \& Cruz, S. (2014). Os jogos mais jogados pelos alunos do Ensino Básico ao Ensino Superior.. In A. A. Carvalho, S. Cruz, C. G. Marques, A. Moura, \& I. Santos (Eds.), Atas do 2. ${ }^{\circ}$ Encontro sobre Jogos e Mobile Learning (pp. 23-37). Braga: CIEd. Retrieved from http://hdl.handle.net/10316/25740 
Carr, N. (2012). Os Superficiais - O que a Internet está a fazer aos nossos cérebros. Lisboa: Gradiva.

Connolly, T. M., Boyle, E. A., MacArthur, E., Hainey, T., \& Boyle, J. M. (2012). A systematic literature review of empirical evidence on computer games and serious games. Computers \& Education, 59(2), 661-686. doi:10.1016/j.compedu.2012.03.004

Csikszentmihalyi, M. (2002). Fluir. Sta Maria da Feira: Relógio D’Água.

Fitz-Walter, Z., Tjondronegoro, D., \& Wyeth, P. (2012). A gamified mobile application for engaging new students at university orientation. In Proceedings of the 24th Australian Computer-Human Interaction Conference on - OzCHI '12 (pp. 138-141). New York, New York, USA: ACM Press. doi:10.1145/2414536.2414560

Gee, J. P. (2003). What Video Games have to teach us about learning and literacy. EUA: Palgrave Macmillan.

Gee, J. P. (2007). Good Video Games + Good Learning: Collected Essays on Video Games, Learning and Literacy. New York: Peter Lang.

Hamari, J., \& Nousiainen, T. (2015). Why Do Teachers Use Game-Based Learning Technologies? The Role of Individual and Institutional ICT Readiness. In Proceedings of the 48th Annual Hawaii International Conference on System Sciences (HICSS). Hawaii: IEEE.

Hamari, J., Shernoff, D. J., Rowe, E., Coller, B., Asbell-Clarke, J., \& Edwards, T. (2016). Challenging games help students learn: An empirical study on engagement, flow and immersion in game-based learning. Computers in Human Behavior, 54, 170-179. doi:10.1016/j.chb.2015.07.045

Huizinga, J. (2000). Homo Ludens ( $4^{\mathrm{a}}$ edição). São Paulo: Editora Perspectiva S.A.

Kapp, K. M. (2012). The Gamification of Learning and Instruction: Game-based methods and strategies for training and education. San Francisco: Pfeiffer.

Klopfer, E. (2008). Augmented Learning: Research and design of mobile educational games. Cambridge, Massachusetts: MIT Press.

Koster, R. (2005). The Theory of Fun for Game Design. EUA: Paraglyph Press.

Laurillard, D. (2002). Rethinking University Teaching. A conversational framework for the effective use of learning technologies. London: Routledge Falmer.

Malliet, S., \& Meyer, G. de. (2005). The History of the Video Game. In J. Raessens \& J. Goldstein (Eds.), Handbook of Computer Game Studies (pp. 25-45). London: The MIT Press.

Martins, A. I., Queirós, A., Rocha, N. P., \& Santos, B. S. (2013). Avaliação de Usabilidade: Uma Revisão Sistemática da Literatura. Revista Ibérica de Sistemas E Tecnologias de Informação, 11, 31-43. doi:10.4304/risti.11.31-43

McGonigal, J. (2011). Reality is broken - Why games make us better and how they can change the world. New York: Penguin Books. 
Oliveira, R. (2014). Aplicação de audience response systems em aulas de biologia molecular como ferramenta de incentivo à participação ativa nas aulas. In A. A. Carvalho, S. Cruz, C. G. Marques, A. Moura, \& I. Santos (Eds.), Atas do 2.o Encontro sobre Jogos e Mobile Learning (pp. 444-451). Braga: CIEd. Retrieved from http://hdl.handle.net/10316/25740

Poy-Castro, R., Mendaña-Cuervo, C., \& González, B. (2015). Diseño y evaluación de un juego serio para la formación de estudiantes universitarios en habilidades de trabajo en equipo. Revista Ibérica de Sistemas E Tecnologias de Informação, E3, 71-83. doi:10.17013/risti.e3.71-83

Prensky, M. (2010). Teaching Digital Natives. Partnering for real learning. Thousand Oaks: Corwin.

Salen, K., \& Zimmerman, E. (2004). Rules of Play - Game Design Fundamentals. Toxicological sciences: an official journal of the Society of Toxicology (Vol. 138). Massachusetts London, England: The MIT Press Cambridge. doi:10.1093/toxsci/ kft290

Sherry, J. L., Lucas, K., Greenberg, B. S., \& Lachlan, K. (2006). Video game uses and gratifications as predictors of use and game preference. In P. Vorderer \& J. Brryant (Eds.), Playing Computer games: Motivations, responses, and consequences (pp. 248-262). Mahwah: Erlbaum.

Squire, K. D. (2011). Video Games and Learning - Teaching and Participatory Culture in the digital age. New York: Teachers College, Columbia University.

Whitton, N. (2009). Learning and Teaching with Computer Games in Higher Education. In T. Connolly, M. Stansfield, \& L. Boyle (Eds.), Games-Based Learning for Advancements for Multi-Sensory Human Computer Interfaces: Techniques and Effective Practice (pp. 18-33). Hershey, PA: Information Science Reference. doi:10.4018/978-1-60566-360-9.choo2

Whitton, N. (2010). Learning with Digital Games: A Practical Guide to Engaging Students in Higher Education. New York: Routledge.

Zolotov, M. J. N. (2014, February 29). Collecting data for indoor mapping of the university of Münster via a location based game. Universität Münster. Retrieved from http://run.unl.pt/handle/10362/11754 